\title{
MODELAGEM MATEMÁTICA DO ESCOAMENTO DE FLUIDOS EM TUBOS HELICOIDAIS
}

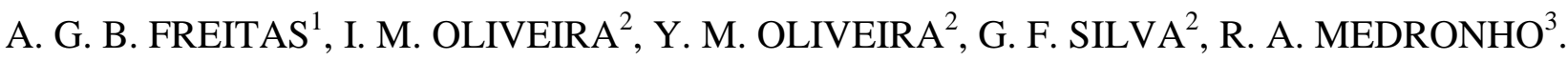 \\ ${ }^{1}$ Universidade Federal do Rio de Janeiro, Escola de Química, Programa de Pós-Graduação em \\ Tecnologia de Processos Químicos e Bioquímicos. \\ ${ }^{2}$ Universidade Federal de Sergipe, Núcleo de Engenharia de Petróleo. \\ ${ }^{3}$ Universidade Federal do Rio de Janeiro, Escola de Química, Dept. de Engenharia Química. \\ E-mail para contato: andreafreitas@eq.ufrj.br.
}

\begin{abstract}
RESUMO - O tubo helicoidal é, há muito tempo, empregado em trocas térmicas e é, atualmente, objeto de pesquisa na área de separação de fluidos e particulados. Neste estudo, o objetivo é modelar e caracterizar de forma preliminar como se desenvolve o escoamento monofásico de fluidos em tubos helicoidais. Este é um estudo precursor na descrição do escoamento secundário, o qual é o processo responsável pela separação de dois fluidos imiscíveis com densidades diferentes. Uma dessas aplicações é o tratamento de água produzida, visando à separação das gotas de óleo da água. Assim, foram empregadas as equações de Navier-Stokes e equações básicas referentes às características de um tubo helicoidal, baseadas nos estudos de Dean (1927) e Germano (1989) e sem transferência de calor no processo. Com isso, podem ser esclarecidos quais os melhores parâmetros, por exemplo, a curvatura e a torção, que melhor se aplicam à separação óleo-água.
\end{abstract}

\section{INTRODUÇÃO}

A análise de escoamento em tubos helicoidais teve início com Eustice (1911) e continuou com os estudos realizados por Dean (1927) em tubos toroidais, o qual incluiu os conceitos de curvatura e de escoamento secundário. Posteriormente, Germano $(1982,1989)$ aperfeiçoou os conceitos anteriores acrescentando o conceito de torção e a coordenada ortogonal.

A modelagem parte do princípio que a natureza dos fenômenos físicos de um determinado processo deve ser entendida, determinando quais grandezas físicas atuam no sistema e como elas o afetam. Isso pode ser realizado através de ensaios experimentais em laboratório ou por modelos matemáticos (Coelho, 2011).

Para se descrever um fluido escoando em um tubo helicoidal, deve-se considerar, dentre outros, a influência do campo centrífugo e as equações de Navier-Stokes. A complexidade do escoamento em tubos helicoidais é grande, principalmente para elevados números de Reynolds, pelo surgimento de um escoamento secundário (Huttl, 1999). Com isso, simulações utilizando fluidodinâmica computacional têm sido largamente empregadas para resolver as equações de conservação visando melhor observar melhor o fenômeno.

Segundo Yu et al. (2003), a razão de curvatura e o passo são parâmetros chaves que afetam o campo centrífugo atuando no fluido em um tubo helicoidal. Quanto maior o raio de curvatura, 


\section{9 a 22 de outubro de 2014 \\ Florianópolis/SC}

maior a força inercial centrífuga ou, por simplicidade, força centrífuga; e quanto maior o passo, maior o efeito de torção que causa uma redução na força centrífuga. Eles obtiveram boa concordância entre os dados experimentais (Laser Doppler Anemometry - LDA) e os resultados numéricos (Computational Fluid Dynamics - CFD).

O objetivo do presente trabalho é modelar e caracterizar de forma preliminar como se desenvolve o escoamento monofásico em tubos helicoidais, além de analisar a influência da torção e curvatura; sendo assim, um estudo preliminar na descrição do escoamento secundário, que é o processo fundamental para a separação de dois fluidos de densidades aparentes diferentes. Uma possível aplicação é o tratamento de água produzida em um tubo helicoidal, onde se pretende que ocorra a separação do óleo da água.

\section{METODOLOGIA}

Inicialmente foram definidas quais as condições a serem trabalhadas, sendo adotadas as de fluido newtoniano e incompressível, regime permanente, processo adiabático, fluxo tridimensional e desprezo da influência do campo gravitacional. Houve também preferência pelos baixos números de Reynolds, além da consideração de que o fluido é um meio contínuo.

Por existirem diversos estudos já publicados referentes ao escoamento em tubos helicoidais baseados nos estudos de Dean e Germano, foi realizada uma revisão dos trabalhos que se encaixam nas condições escolhidas. Como estes trabalhos abordam a modelagem matemática de um tubo helicoidal, pôde-se comparar os melhores resultados de coordenadas aplicadas às equações de Navier-Stokes, além da definição de parâmetros básicos que definem um tubo helicoidal.

\section{RESULTADOS E DISCUSSÃO}

\subsection{Equações Básicas}

Alguns autores, como Austin (1973) e Elmaleh e Jabbouri (1991), ambos apud Pelissari (2006), mostram que nos escoamentos em tubos helicoidais, o número de Reynolds crítico (Rec) pode ser até quatro vezes maior que em tubos retos, onde Rec é o limite do regime de escoamento laminar. De acordo com Ito (1959) apud Pelissari (2006), o valor de Rec, para escoamentos em tubos helicoidais pode ser dado pela relação apresentada na Equação 1.

$$
\operatorname{Rec}=20000\left(\frac{d}{D}\right)^{0,32} \text { para } 0,016<\left(\frac{d}{D}\right)<0,067
$$

Onde Rec é o número de Reynolds crítico, d é o diâmetro do tubo (m) e D o diâmetro da hélice $(\mathrm{m})$.

Algumas faixas de valores de $\frac{d}{D}$ são estudadas em outros trabalhos citados por Pelissari (2006).

Mishra e Gupta (1979) utilizaram a Equação 1 mas com um limite diferente, na razão dos diâmetros. Eles estabeleceram uma restrição ao espaço entre voltas, $\left(\frac{H}{D}\right)$, como pode ser visto na 
Equação 2 fornecida por Srinivasan et al. (1968). Nesta equação, H é a distância entre as voltas e $\mathrm{D}$ o diâmetro da hélice.

$$
\operatorname{Rec}=2100\left[1+12\left(\frac{d}{D}\right)^{0,28}\right] \quad 0,003<\left(\frac{d}{D}\right)<0,15 ; 0<\left(\frac{H}{D}\right)<25,4
$$

Ko e Ting (2004), empregando uma faixa mais ampla de $\frac{d}{D}, 0,01<\left(\frac{d}{D}\right)<0,3$, obtiveram a Equação 3.

$$
\operatorname{Rec}=2100\left[1+12\left(\frac{d}{D}\right)^{0,5}\right]=\operatorname{Re}_{H}
$$
estudo.

O Reynolds crítico citado acima foi definido como Reynolds helicoidal $\left(\mathrm{Re}_{\mathrm{H}}\right)$ no presente

Para dar continuidade à caracterização do escoamento secundário e a dimensão de um tubo helicoidal é necessário definir mais alguns parâmetros, que são mostrados nas Equações de 4 a 10. Primeiramente é definida a distância entre as voltas da hélice, que é fornecido por:

$$
H=2 \pi \cdot P \rightarrow P=\frac{H}{2 \pi}
$$

Onde H é a distância entre as voltas da hélice (m) e P é o passo da hélice (m).

Para definir o número de Dean, primeiro devem-se conhecer os parâmetros curvatura (k) e curvatura adimensional $(\varepsilon)$, fornecidos como:

$$
k=\frac{R}{R^{2}+P^{2}}
$$

Onde k é a curvatura $\left(\mathrm{m}^{-1}\right)$, R é o raio da hélice (m) e P é o passo da hélice (m).

$$
\varepsilon=k \cdot r^{\prime}
$$

Onde $\varepsilon$ é a curvatura adimensional, k é a curvatura $\left(\mathrm{m}^{-1}\right)$ e $\mathrm{r}^{\prime}$ é o raio do tubo $(\mathrm{m})$. O número de Dean é, então:

$$
D e=\left(\varepsilon^{0,5}\right) \cdot R e
$$

Onde De é número de Dean e Re o número de Reynolds convencional.

Berger et al. (1983) mostraram que o limite superior do número de Dean para o regime de escoamento laminar em tubos helicoidais é aproximadamente 5.000.

Para a definição do número de Germano é necessário conhecer a torção, fornecida como:

$$
\tau=\frac{P}{R^{2}+P^{2}}
$$


Onde $\tau$ é a torção $\left(\mathrm{m}^{-1}\right)$, P é o passo da hélice $(\mathrm{m})$ e R é o raio da hélice (m).

Germano $(1982,1989)$ aplicou as equações de Dean (1928) no escoamento num tubo helicoidal, e demonstrou que estas dependem não somente do número de Dean, mas também de um novo parâmetro, a torção adimensional $(\lambda)$, fornecido por:

$$
\lambda=\frac{\tau}{k}
$$

Onde $\lambda$ é a torção adimensional, $\tau$ é a torção $\left(\mathrm{m}^{-1}\right)$ e $\mathrm{k}$ é a curvatura $\left(\mathrm{m}^{-1}\right)$.

$$
G n=\tau \cdot r \cdot R e_{H}
$$

Onde Gn é o número de Germano, $\tau$ é a torção $\left(\mathrm{m}^{-1}\right)$, r é o raio do tubo (m) e $\operatorname{Re}_{\mathrm{H}}$ é o número de Reynolds Helicoidal, já definido na Equação 3.

As comparações entre as equações e os resultados apresentados em diferentes trabalhos são em alguns casos difíceis e complexos. Há sistemas de coordenadas e aproximações de ordens diferentes que têm sido recentemente adotadas nos diversos estudos de escoamentos em tubos helicoidais.

\subsection{Equações de Navier-Stokes}

As equações de Navier-Stokes em coordenadas ortogonais foram utilizadas em diversos trabalhos, como Tutlle (1990) e Kao (1987) apud Germano (1989). No entanto, dependendo do autor, a simbologia para cada termo das equações é diferenciado. Com isso, a interpretação utilizada neste trabalho foi feita a partir da publicação de Huttl (1999), por este apresentar fácil entendimento dos termos, além de representações mais atuais dos mesmos.

Germano (1989) realizou uma extensão das equações de Dean com o objetivo de introduzir a torção como parâmetro do escoamento de um tubo helicoidal. Para isso ele utilizou coordenadas ortogonais, de modo que se $\tau$ (torção) for zero seu sistema de coordenadas volta a ser o nãoortogonal utilizado por Dean (1927).

Uma das desvantagens dada por Gammack e Hydon (2001) a respeito desta coordenada é de que ela se refere ao centro do tubo. Então, ela não contém o campo vetorial tangente às linhas de congruência da hélice para um passo fixo. Isto inibe o fornecimento de um meio natural para distinguir entre os componentes axiais e secundários de um escoamento. Entretanto, segundo Tuttle (1990) esta coordenada evita o problema de falsas interpretações dos vetores de velocidade covariantes e contravariantes, que são obtidos nas soluções da coordenada não-ortogonal. Problema este enfrentado por Wang (1981) apud Tuttle (1990) e Murata et al. (1981) apud Tuttle (1990), que obtiveram diferentes resultados por interpretarem de forma diferente estes vetores.

Germano $(1982,1989)$ introduziu o fato de que um sistema de coordenadas helicoidal pode ter como referência o sistema de coordenadas cartesianas. Aplicando as coordenadas helicoidais para a direção axial, $r$ para a direção radial e $\theta$ para a direção angular, a posição para qualquer ponto X, interno ao tubo helicoidal pode ser descrito pelo vetor $\vec{X}$ (Huttl, 1999), fornecido pela Equação 11. 


$$
\vec{X}=\vec{P}(s)-r \operatorname{sen}(\theta-\tau S) \vec{N}(s)+r \cos \vec{B}(s)
$$

Onde $\vec{P}, \vec{N}$ e $\vec{B}$ são as direções tangencial, normal e binormal de uma curva genérica no eixo do tubo no ponto considerado. A coordenada utilizada está ilustrada na Figura 1.

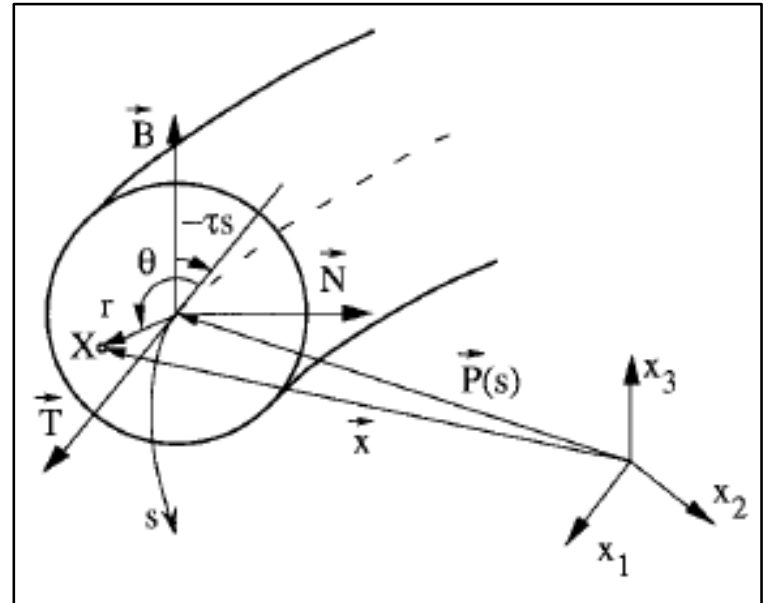

Figura 1 - Esquema do sistema de coordenadas helicoidal ortogonal (s, r, $\theta)$, conforme Germano (1982,1989). Fonte: Huttl (1999).

O sistema de coordenadas helicoidal ortogonal é apresentada na Equação 12.

$$
d \vec{x} \cdot d \vec{y}=(1+k r \operatorname{sen}(\theta-\tau s))^{2} d s^{2}+d r^{2}+r^{2} d \theta^{2}
$$

Onde ds, dr e d $\theta$ são incrementos infinitesimais nas direções axial, radial e angular. Desta forma são obtidos os fatores escalares $h_{s}, h_{r}$ e $h_{\theta}$, que são utilizados para expressar as equações de Navier-Stokes em coordenadas helicoidais. Estes fatores são apresentados nas Equações 13 a 15.

$$
\begin{aligned}
& h_{S}=1+k r \operatorname{sen}(\theta-\tau s) \\
& h_{r}=1 \\
& h_{\theta}=r
\end{aligned}
$$

As Equações de Navier-Stokes escritas de acordo com o sistema de coordenadas helicoidal ortogonal são demostradas nas Equações 16 a 19.

Equação da Conservação da Massa

$\frac{\partial}{\partial s}\left(r u_{s}\right)+\frac{\partial}{\partial r}\left(h_{s} r u_{r}\right)+\frac{\partial}{\partial \theta}\left(h_{s} u_{\theta}\right)=0$

Momento s

$$
\frac{\partial u_{s}}{\partial t}+\frac{1}{h_{s}} \frac{\partial}{\partial s}\left(u_{s} u_{s}\right)+\frac{1}{h_{s} r} \frac{\partial}{\partial r}\left(h_{s} r u_{s} u_{r}\right)+\frac{1}{h_{s} r} \frac{\partial}{\partial \theta}\left(h_{s} u_{s} u_{\theta}\right)+\frac{k \operatorname{sen}(\theta-\tau s)}{h_{s}} u_{s} u_{r}+
$$


$\frac{k \cos (\theta-\tau s)}{h_{s}} u_{s} u_{\theta}=\frac{1}{h_{s}} \frac{\partial p}{\partial s}+\frac{1}{R e_{\tau}}\left[\frac{2}{h_{s}} \frac{\partial}{\partial s}\left(\frac{1}{h_{s}}\left(\frac{\partial u_{s}}{\partial s}+k \operatorname{sen}(\theta-\tau s) u_{r}+k \cos (\theta-\right.\right.\right.$

$\left.\left.\tau s) u_{\theta}\right)\right)+\frac{1}{h_{s} r} \frac{\partial}{\partial r}\left(h_{s} h_{s} r \frac{\partial}{\partial r}\left(\frac{u_{s}}{h_{s}}\right)+r \frac{\partial u_{r}}{\partial s}\right)+\frac{1}{h_{s} r} \frac{\partial}{\partial \theta}\left(\frac{h_{s} h_{s}}{r} \frac{\partial}{\partial \theta}\left(\frac{u_{s}}{h_{s}}\right)+\frac{\partial u_{\theta}}{\partial s}\right)+$

$\left.k \operatorname{sen}(\theta-\tau s)\left(\frac{\partial}{\partial r}\left(\frac{u_{s}}{h_{s}}\right)+\frac{1}{h_{s} h_{s}} \frac{\partial u_{r}}{\partial s}\right)+k \cos (\theta-\tau s)\left(\frac{1}{r} \frac{\partial}{\partial \theta}\left(\frac{u_{s}}{h_{s}}\right)+\frac{1}{h_{s} h_{s}} \frac{\partial u_{\theta}}{\partial s}\right)\right]$

Momento r

$$
\begin{aligned}
& \frac{\partial u_{r}}{\partial t}+\frac{1}{h_{s}} \frac{\partial}{\partial s}\left(u_{s} u_{r}\right)+\frac{1}{h_{s} r} \frac{\partial}{\partial r}\left(h_{s} r u_{r} u_{r}\right)+\frac{1}{h_{s} r} \frac{\partial}{\partial \theta}\left(h_{s} u_{r} u_{\theta}\right)-\frac{k \operatorname{sen}(\theta-\tau s)}{h_{s}} u_{s} u_{s}-\frac{u_{\theta} u_{\theta}}{r}= \\
& -\frac{\partial p}{\partial r}+\frac{1}{R e_{\tau}}\left[\frac{1}{h_{s}} \frac{\partial}{\partial s}\left(h_{s} \frac{\partial}{\partial r}\left(\frac{u_{s}}{h_{s}}\right)+\frac{1}{h_{s}} \frac{\partial u_{r}}{\partial s}\right)+\frac{2}{h_{s} r} \frac{\partial}{\partial r}\left(h_{s} r \frac{\partial u_{r}}{\partial r}\right)+\frac{1}{h_{s}} \frac{\partial}{\partial \theta}\left(h _ { s } \left(\frac{1}{r r} \frac{\partial u_{r}}{\partial \theta}+\right.\right.\right. \\
& \left.\left.\frac{\partial}{\partial r}\left(\frac{u_{\theta}}{r}\right)\right)\right)-\frac{2 k \operatorname{sen}(\theta-\tau s)}{h_{s} h_{s}}\left(\frac{\partial u_{s}}{\partial s}+k \operatorname{sen}(\theta-\tau s) u_{r}+k \cos (\theta-\tau s) u_{\theta}\right)-\frac{2}{r r}\left(\frac{\partial u_{\theta}}{\partial \theta}+\right. \\
& \left.\left.u_{r}\right)\right]
\end{aligned}
$$

Momento $\theta$

$$
\begin{aligned}
& \frac{\partial u_{\theta}}{\partial t}+\frac{1}{h_{s}} \frac{\partial}{\partial s}\left(u_{s} u_{\theta}\right)+\frac{1}{h_{s} r} \frac{\partial}{\partial r}\left(h_{s} r u_{r} u_{\theta}\right)+\frac{1}{h_{s} r} \frac{\partial}{\partial \theta}\left(h_{s} u_{\theta} u_{\theta}\right)-\frac{k \cos (\theta-\tau s)}{h_{s}} u_{s} u_{s}+ \\
& \frac{1}{r} u_{r} u_{\theta}=-\frac{1}{r} \frac{\partial p}{\partial \theta}+\frac{1}{R e_{\tau}}\left[\frac{1}{h_{s}} \frac{\partial}{\partial s}\left(\frac{h_{s}}{r} \frac{\partial}{\partial \theta}\left(\frac{u_{s}}{h_{s}}\right)+\frac{1}{h_{s}} \frac{\partial u_{\theta}}{\partial s}\right)+\frac{1}{h_{s} r} \frac{\partial}{\partial r}\left(h_{s}\left(\frac{\partial u_{r}}{\partial \theta}+r r \frac{\partial}{\partial r}\left(\frac{u_{\theta}}{r}\right)\right)\right)+\right. \\
& \frac{2}{h_{s} r r} \frac{\partial}{\partial \theta}\left(h_{s}\left(\frac{\partial u_{\theta}}{\partial \theta}+u_{r}\right)\right)-\frac{2 k \cos (\theta-\tau s)}{h_{s} h_{s}}\left(\frac{\partial u_{s}}{\partial s}+k \operatorname{sen}(\theta-\tau s) u_{r}+k \cos (\theta-\tau s) u_{\theta}\right)+ \\
& \left.\left(\frac{1}{r r} \frac{\partial u_{r}}{\partial \theta}+\frac{\partial}{\partial r}\left(\frac{u_{\theta}}{r}\right)\right)\right]
\end{aligned}
$$

Estas equações são escritas na forma adimensional e a densidade de massa adimensional é definida como 1 . Onde $\mathrm{u}_{\mathrm{r}} \mathrm{u}_{\theta}$ e $\mathrm{u}_{\mathrm{s}}$ são as componentes da velocidade nas direções $\mathrm{r}, \theta$ e $\mathrm{s}$, respectivamente, k é a curvatura, $\tau$ a torção, $\mathrm{R}$ o raio do tubo e o $\mathrm{Re}_{\tau}$ nota-se na Equação 20 .

$$
R e_{\tau}=\frac{R u_{\tau}}{V}
$$

Onde $u_{\tau}$ é a velocidade de cisalhamento média é definida na Equação 21.

$$
\mathrm{u}_{\tau}=\sqrt{\frac{\tau_{\mathrm{w}, \mathrm{m}}}{\rho}}
$$

Onde $\rho$ é a massa específica e $\tau_{\mathrm{w}, \mathrm{m}}$ é apresentada na Equação 22.

$$
\tau_{w, m}=\frac{1}{2 \pi} \int_{\theta=0}^{2 \pi} \tau_{w}(\theta) d \theta
$$

$\mathrm{O} \mathrm{R}$, a $u_{\tau}$ e o $t_{r e f}=R / u_{\tau}$ são usados como variáveis de escala. 
24.

Os números de Dean e Germano baseados nesta escala são dadas pelas Equações 23 e

$$
\begin{aligned}
& D e_{\tau}=\sqrt{k} R e_{\tau} \\
& G n_{\tau}=\tau R e_{\tau}
\end{aligned}
$$

\subsection{Discussão}

Como o objetivo deste trabalho foi descrever as equações do comportamento de um fluido dentro de um tubo helicoidal, o balanço coerente com este objetivo são os balanços diferencias, os quais foram expressos no tópico das equações de Navier-Stokes. Por isso, optou-se por não realizar os balanços globais de massa e de quantidade de movimento.

Através da resolução das equações de Navier-Stokes é possível obter valores de torções e curvaturas eficazes no surgimento de um escoamento secundário. Como sugestão estas resoluções podem ser feitas aplicadas em programas de fluidodinâmica computacional ou de soluções numéricas como o DNS ou LED.

Com respeito à torção, Sartori (2006) mostrou que seu efeito físico no padrão de escoamento é menos pronunciado do que o efeito da curvatura. Porém, devido a sua influência no escoamento secundário, que conduz a um aumento nas flutuações de energia cinética e da taxa de dissipação, este parâmetro não pode ser negligenciado. Seu principal efeito é aumentar a amplitude das componentes radiais e circunferenciais da velocidade e, consequentemente, seus gradientes espaciais, ajudando assim a entender porque a energia cinética turbulenta é aumentada quando a torção é aplicada. Outro efeito da torção observado é a introdução de forças de inércia adicionais.

Germano (1987) concluiu que o efeito da torção tende a aumentar à medida que número de Reynolds diminui e confirmou o fato de que para um tubo helicoidal, a torção não tem um efeito primário no escoamento secundário. Isto foi comprovado quando a equação do escoamento secundário não mostrava dependência de primeira ordem com a torção.

Uma boa fonte de várias pesquisas desenvolvidas nessa área podem ser encontradas nos trabalho de Alam et al. (2007), Gammack e Hydon (2001) e Berger et al. (1983).

\section{CONCLUSÃO}

Um estudo preliminar do escoamento monofásico em um tubo helicoidal foi realizado com êxito, assim como uma breve descrição da importância da torção e curvatura no estabelecimento de um escoamento secundário. Este trabalho será aplicado em estudos futuros acerca do escoamento bifásico para o tratamento de água produzida, aproveitando-se do escoamento secundário que separará as fases presentes, água e óleo. 


\section{REFERÊNCIAS}

ALAM, M. M.; OTA, M.; FERDOWS, M.; ISLAMV, M. N.; WAHIDUZZAMAN, M., YAMAMOTO, K. Flow through a rotating helical pipe with a wide range of the Dean number. Arch. Mech., 59, 6, pp. 501-517, Warszawa 2007.

BERGER, S. A.; TALBOT, L.; YAO, L. S. Flow in curved pipes. Annual Reviews Fluid Mech., 15:461-512, 1983.

COELHO, D. B. Desempenho de um hidrociclone para separação de águas oleosas. Monografia (Graduação) - Universidade Federal do Rio Grande do Norte. Centro de Tecnologia. Departamento de Engenharia Química. Natal, 2011.

DEAN, W. R. Note on the motion of fluid in a curved pipe. Philosophical Magazine, 4(7), pp. 208-223, 1927.

EUSTICE, J. Flow of water in curved pipes. Proc. R. Soc. Lond. Ser. A, 84, 107-118, 1910.

GAMMACK, D.; HYDON, P. E. Flow in pipes with non-uniform curvature and torsion. J. Fluid Mech., vol. 433, pp. 357-382, 2001.

GERMANO, M. On the effect of torsion on a helical pipe. J. Fluid Mech., vol. 125, pp. 1-8, 1982.

GERMANO, M. The Dean equations extended to a helical pipe flow. J. Fluid Mech., vol. 203, pp. 289-305, 1989.

HUTTL, T. J.; WAGNER, C.; FRIEDRICH, R. Navier-Stokes Solutions of Laminar Flows Based on Orthogonal Helical co-ordinates. Int. J. Numer. Meth. Fluids 29: pp. 749-763 (1999).

PELISSARI, L. M. T. Influência de parâmetros de projeto no desempenho do floculador tubular helicoidal aplicado ao tratamento de água de abastecimento. Vitória, 2006. 119p. Dissertação (Mestrado em Engenharia Ambiental) - Universidade Federal do Espírito Santo.

SARTORI, M. Caracterização Hidrodinâmica de Floculadores Tubulares Helicoidais por Meio de Simulação Numérica Tridimensional. Vitória, 2006. 106p. Dissertação (Mestrado em Engenharia Ambiental) - Universidade Federal do Espírito Santo.

TUTTLE, E. R. Laminar flow in twisted pipes. J. Fluid Mech., 219, pp. 545-570, 1990.

YU, B.; ZHENG, B.; LIN, C. X.; PEÑA, O. J.; EBADIAN, M. A. Laser Doppler anemometry measurements of laminar flow in helical pipe. Experimental Thermal and Fluid Science V. 27 pp. 855-865, 2003. 